\title{
Helligkeitsänderungen der Planeten (56) Melete und (71) Niobe.
}

\author{
Von G. Miuller.
}

In Nr. 1359 der Astr. Nachr. sind von Herrn Prof. Tietjen zwei über längere Zeiträume ausgedehnte Reihen von Grössenschätzungen der Planeten Melete und Niobe veröffentlicht worden. Da die auf mittlere Opposition reducirten Grössenangaben in beiden Fällen einen deutlichen Gang erkenden lassen, so schien es mir von Interesse zu untersuchen, ob die Intensitätsänderungen ebenso wie bei : den von mit und Parkhurst photometrisch beobachteten $i$ Asteroiden mit den entsprechenden Phasenvariationen im Zusammenhang stünden. Ich erlaube mir die Resultate ' dieser Untersuchung, welche einen derartigen Zusammen- ! hang in der That zweifellos feststellen und einen höchst erwtinschten Beitrag zur Beurtheilung des photometrischen! Verhaltens der kleinen Planeten liefern, hier mitzutheilen.

In der folgenden Zusammenstellung sind in den ersten, beiden Columnen das Beobachtungsdatum und die direct geschätzten Grössen angegeben. Die dritte Columne enthält die auf mittlere Oppósition reducirten Grössen, welche von den in Nr. 1359 angefuhrten Werthen zum Theil um geringe Beträge abweichen, weil bei der Neuberechnung der Reductionen die neuesten Elemente der Planeten zu Grunde gelegt worden sind. Die vierte Columne giebt den Phasenwinkel an, d. h. den Winkel an Planeten im Dreieck: Sonne-Planet - Erde.

Die letzte Beobachtung von Niobe habe ich leider unberucksichtigt lassen mussen, weil in den a. a. O. nitgetheilten Zahlen offenbar ein Irrthum vorliegt. Das Datum Oct. 23 stimmt gar nicht zu der von Tietjen benutzten Reduction auf mittlere Opposition; vielleicht soll es Dec. 23 ! statt Oct. 23 heissen.

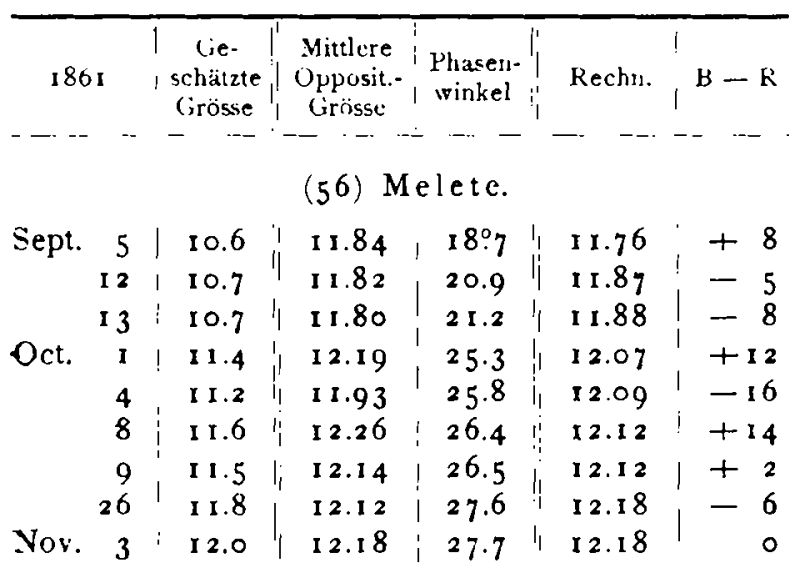

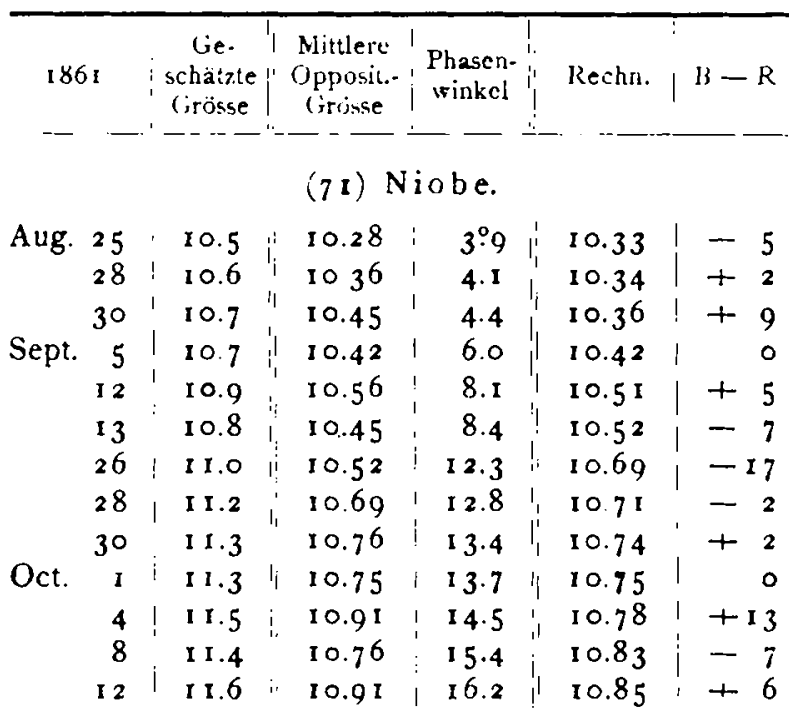

Aus beiden Reihen geht übereinstimmend hervor, dass die Lichtstärke mit wachsenden Phasenwinkeln geringer wird. Nimmt man den Gang proportional der Phasenänderung an und legt den Beobachtungen die einfache Formel : $h==h_{0}+b \alpha$ zu Grunde, worin $h_{0}$ die Grösse bei voller Beleuchtung und $b$ die Aenderung für $I^{\circ}$ 'hasenwinkel ausdrückt, so giebt die Auflösung nach der Methode der kleinsten Quadrate die Gleichungen:

$$
\begin{array}{ll}
\text { tür Melete }: & h=10.90+0.0462 \alpha \\
\text {, Niobe }: & h==10.17+0.0422 \alpha
\end{array}
$$

Mit Hülfe dieser Formeln sind die in der vorletzten Columne der obigen Zusammenstellung angeführten Werthe berechnet, und in der letzten Columne sind die Unterschiede zwischen Beobachtung und Rechnung in Hundertstel Grössenclassen mitgetheilt. Aus diesen Werthen geht erstens hervor, dass die Tietjen'schen Grössenschätzungen eine ganz aussergewöhnliche Sicherheit besitzen und zweitens, dass die Reobachtingen in durchaus befriedigender Weise durch die abgeleiteten Formeln dargestellt werden.

Was die gefundenen Phasencoefficienten anbetrifft, so liegen dieselben innerhalb der Grenzen, welche durch meine und Parkhurst's photometrische Messungen bisher für die kleinen Planeten festgestellt worden sind. Bei den von mir untersuchten 17 Asteroiden (Potsdamer Publicationen, 
Band 8 , Seite 364 ) schwanken die Coefficienten zwischen 0.018 und 0.042 , und wenn man von den 36 Parkhurst' schen Asteroiden (Annals of the Harvard College Observatory, Vol. 29, p. 86) alle diejenigen unberuicksichtigt lässt, bei denen die Bestimmung wegen der Kleinheit des benutzten Phasenintervalls ganz unsicher ist, so bewegen sich die Coefficienten zwischen 0.016 und 0.053 . Die beiden Planeten Melete und Niobe passen also hinsichtlich ihres photometrischen Verhaltens in diese Reihen hinein, und man wird daher die Tietjen'schen Beobachtungen als eine weitere Bestätigung dafür ansehen dürfen, dass bei den Helligkeitsbestimmungen der kleinen Planeten die Reductionen wegen Phasenbeleuchtung keineswegs zu vernachlässigen sind. Daraus folgt aber, wie ich bereits an anderer Stelle betont habe, dass die bisher angenommenen mittleren Oppositions. helligkeiten der Asteroiden im Allgemeinen zu klein sind, weil die Schätzungen, auf denen z. B. die Angaben im Berl. Astr. Jahrb. beruhen, meistens nicht bei voller Beleuchtung, sondern bei allen möglichen Phasen angestellt sind. Für Melete und Niobe giebt das Jahrbuch für 1894 die mittleren Oppositionsgrössen 11.7 und 10.7 an, während nach der obigen Rechnung für volle Beleuchtung die Grössen I0.9 und 10.2 resultiren.

Es wäre mir in hohem Grade erwünscht, wenn mir noch mehr solcher Beobachtungsreihen wie die Tietjen'schen 2u Gebote ständen, und ich würde den Beobachtern der kleinen Planeten zu grossem Dank verpflichtet sein, wenn sie mich auf etwaige schon veröffentlichte Reihen aufmerksam machen oder mir noch nicht publicirte zur Verfuggung stellen woltten, vorausgesetzt dass dieselben ein Phasenintervall von mindestens $10^{\circ}$ umfassten. Gleichzeitig möchte ich die Gelegenheit benutzen, um die Helligkeitsbeobachtungen der Asteroiden für die Zukunft recht warm zu empfehlen. Es eröfinet sich hier noch ein reiches Feld der Thätigkeit, und die weitere Verfolgung der kleinen Himmelskörper gewinnt, von diesem Gesichtspunkt aus betrachtet, wie mir scheint, ein ganz neues Interesse. Wenn die zahlreichen Beobachter der kleinen Planeten die verhältnissmässig geringe Mühe nicht scheuten, mit jeder Positionsbestimmung auch eine Helligkeitsbestimmung zu verbinden und zwar nach der bekannten Argelander'schen Methode durch Stufenvergleichungen mit benachbarten Fixsternen, deren Lichtstärke später durch sorgfältige photometrische Messungen an einer mit geeigneten Instrumenten versehenen Sternwarte ermittelt werden könnte, so würde in Kürze ein reiches Material gesammelt werden können, welches uns nicht nur sichere Auskunft uber das photometrische Ver. halten der Asteroiden geben, sondern vielleicht auch zu manchen interessanten Schlussfolgerungen in Betreff der physischen Beschaffenheit dieser Weltkörper fiihren würde.

Putsdam, Astrophysikalisches Observatorium, 1894 Febr. 28.

G. Müller.

\section{Planet (332) Siri.}

\section{Von A. Berberich.}

Dieser Planet wurde am 19. Mär 1892 von Prof, bewegte. Zwei von Herrn Wolf früher schon ausgemessene M. Wolf auf zwei Platten aufgenommen; weitere Positionen Objecte, die auf andern Platten fehlen, konnten nicht als sind durch die Aufnahmen vom 20. und 22. März gewonnen. | mit dem Planeten identisch betrachtet werden, da die Bahn. Auf einer Photographie vom 25. März ist er dagegen nicht, berechnung auf unmögliche Verhältnisse führte. Herr Wolf sicher zu erkennen, während am 30 . der Planetenstrich, wie, theilte mir jetzt noch die Messungen eines andern, $+18^{s}$ ich mich selbst gelegentlich eines Besuches in Heidelberg und - 4:8 von der Kreisbahnephemeride abstehenden Obuberzeugen konnte, ziemlich deutlich ist. Aus März r 9 und jectes mit, das der Planet gewesen sein kann, wenn freilich 30 ist die in A. N. 3086 angefuhte und in das Berliner diese Identität nicht mit Gewissheit zu behaupten ist. Es Jahrbuch aufgenommene Kreisbahn abgeleitet. Da directe $/$ scheint aber das einzige Object zu sein, das in Frage Beobachtungen nicht vorliegen, so musste zur Berechnung kommen kann. Ich habe es daher benutzt, um eine Bahn einer Ellipse die Planetenposition aus einer Aufnahme vom zu berechnen, der ich aber keine grössere Zuverlässigkeit I9. April entnommen werden, was jedoch mit grossen|zuschreiben möchte als der ersten Kreisbahn.

Schwierigkeiten und erheblicher Unsicherheit verknipft ist, da der Planet sehr schwach war und sich nur noch langsam,

\section{Die Elemente lauten:}

\section{Epoche 1892 April I 9.5 M. 2. Berlin.}

$$
\begin{aligned}
& M=335^{\circ} 40^{\prime} 31^{\prime \prime} \text { i } \\
& \omega=194 \quad 14 \quad 22.2
\end{aligned}
$$

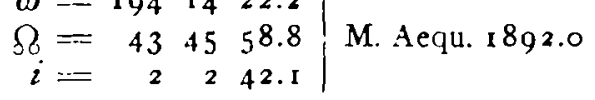

$$
\begin{aligned}
& \varphi==22^{\circ} 7^{\circ} 56^{\prime \prime} .8 \\
& \mu==605^{\prime \prime} 45^{\circ} \\
& \log a==0.51 \times 95^{2} \\
& \text { Mitti. Oppositions-Grösse }=15^{\mathrm{Dt}} \text {. }
\end{aligned}
$$

Vermuthlich ist $\mu$ zu klein und $\varphi$ entsprechend viel zu gross ausgefallen; unter den vorliegenden Verhältnissen bewirken geringe Beobachtungsfehler schon sehr starke Aenderungen der Elemente, die also auf alle Fälle recht unsicher bleiben, bis durch eine Neuauffindung der Planet gesichert werden kann.

Nach Herrn Wolf's Mittheilungen erlaube ich mir, die Planetenörter und die denselben zu Grunde liegenden Messungen anzufuhren. 